\title{
Heating Simulation of Tensile Specimen at Continuous
}

\section{Casting Temperatures}

\author{
Jinmiao $\mathrm{Pu}^{1, \text { a }}$, Qin Qin ${ }^{1, \mathrm{~b}}$, Mingliang Tian ${ }^{1, \mathrm{c}}$, and Xuehui Zhu ${ }^{1, \mathrm{~d}}$ \\ ${ }^{1}$ School of Mechanical Engineering, University of Science and Technology Beijing, \\ Beijing 100083, China. \\ apujinmiao@126.com, 'binqin@me.ustb.edu.cn, ${ }^{\mathrm{c} t m l}$ 817817@163.com, ${ }^{\mathrm{d}}$ senukycc@ \\ 163.com
}

\begin{abstract}
The heating process and heat preservation of the specimens in Gleeble thermal simulation test machine were simulated. A coupled electrical-thermal model is established by ABAQUS. The internal temperature distributions of the specimen were analyzed. The uniform temperature zone of the specimen under the studied conditions is given. The effects of different temperatures and the time of heat preservation were discussed. The influence of temperature distribution on the tensile test was analyzed.
\end{abstract}

Key words: high temperature, tensile specimen, temperature distribution, uniform temperature zone.

\section{Introduction}

Quality standard of the continuous casting slabs is sternly developed to meet the performance requirements. However, there are many defects of the slab appeared during the solidification in the continuous casting process, and these defects are closely related to the high temperature mechanical properties of the slab. In order to improve the quality of the slab, the stress-strain behavior of the slab at high temperature should be studied to obtained its high temperature mechanical properties. Because the temperatures involved are quite high, many researchers tend to use the Gleeble test machine to carry out the tensile test [1-5]. Unfortunately, the specimen's temperature after heating is not uniform in Gleeble, which will have a huge impact on the tensile test. Some investigations have took into account this aspect [6, 7], but the temperatures considered in former were under $800 \mathrm{~K}$ and the latter did not consider the uniform temperature zone.

In present study, the Gleeble tensile specimens in the temperature range of $1273 \mathrm{~K}$ to $1673 \mathrm{~K}$ were simulated by ABAQUS. The distribution of the specimens at high temperature was investigated. Meanwhile, its uniform temperature zone and effects on tensile test were analyzed.

\section{The Establishment of The Model}

\subsection{Geometric Model and Material Parameters}

The heating of the specimen is a thermal-electric coupling problem. Taking into account the used specimen is cylindrical that the diameter is $10 \mathrm{~mm}$ and the length is 
$120 \mathrm{~mm}$, the axisymmetric model is selected. The nuts at both ends of the specimen were ignored in this model. In order to support and protect the area may melt, in the middle of the specimen need to put a quartz glass tube before heating. The contact surfaces between the grips and specimen, the quartz tube and specimen are tied. Three-node linear axisymmetric thermal-electrical coupling triangular element (DCAX3E) is used, as shown in Fig. 1.

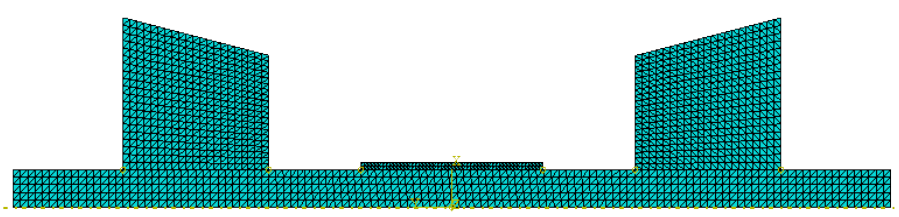

Fig. 1 The model and mesh

The main chemical compositions of AH36 used for the test are as follows: $0.157 \% \mathrm{C}$, 0.25\%Si, 1.1132\%Mn, 0.0162\%P, 0.0044\%S, 0.0375\%Cr, 0.0045\%Mo, 0.0177\%Cu, etc. The thermal and electrical properties are shown in Fig. 2. According to previous studies, the slab of AH36 steel occurs austenite decomposition at about $1273 \mathrm{~K}$ during process of solidification, there are two different trends of heat conductivity on both sides of this point.

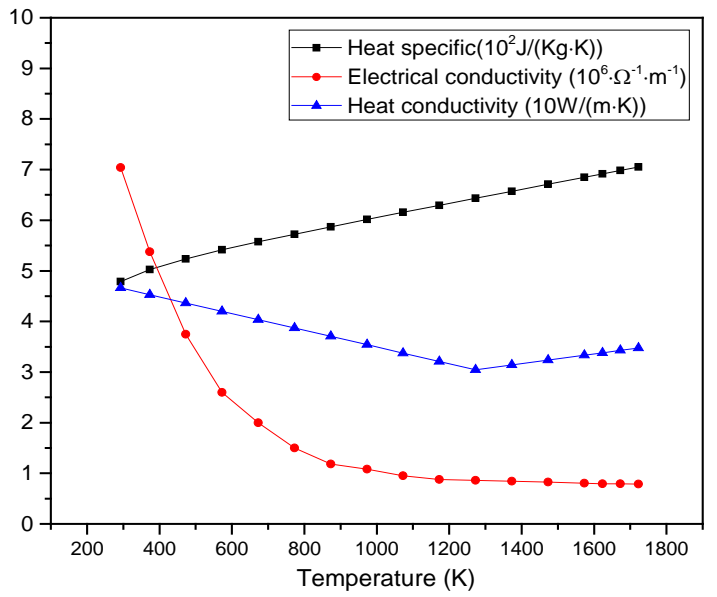

Fig. 2 The thermal and electrical properties of AH36

\subsection{Load and Boundary Conditions}

The specimen was heated by current in Gleeble simulation test machine. In present study, when the load is applied to the model, the uniform surface current is loaded on a grip's boundary, and the boundary of another grip is set to zero potential.

During the heating process, the dissipation of the temperature mainly consists of two parts. Firstly, through the contact conduction of the specimen and grips. In order to keep the test machine work properly, there is continuous cooling by the water pipe on the jaws which directly contact with grips. So the heat transfer coefficient of interface set to $2000 \mathrm{~W} /\left(\mathrm{m}^{2} \cdot \mathrm{K}\right)$. Secondly, there are radiations from surfaces of specimen to vacuum chamber, which the thermal emissivity is 0.8 . 


\section{Results of Temperature Simulation}

\subsection{The Heating Process of Specimen}

Because specimens can be obtained the tissues more similar to the reality of the slab using solidification simulation, the specific heating profile was shown in Fig. 3a. During the process of heating simulation, the current load is gradually adjusted so that the heating profile of the specimen center area is consistent with the predefined. In this job, the time of heat preservation was 2 min (shown in Fig. 3b).
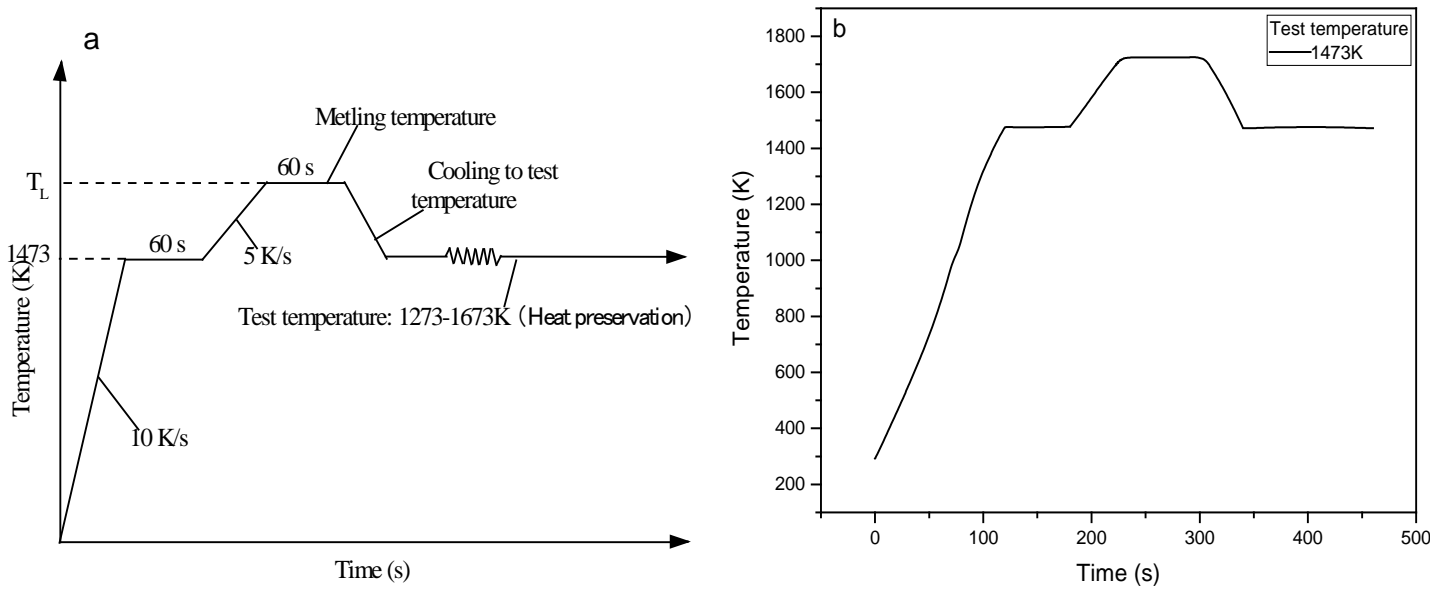

Fig. 3 The heating profile of predefined (a) and simulation (b)

\subsection{Internal Electrical Current and Temperature Distributions of Specimen}

As shown in the red lines of Fig. 4, there are only current densities in the middle of specimen, and the differences of current density on the cross section are very small which the maximum of differences is about $0.4 \%$ of the average. This means that Joule heat generated are substantially equal in the heating section.
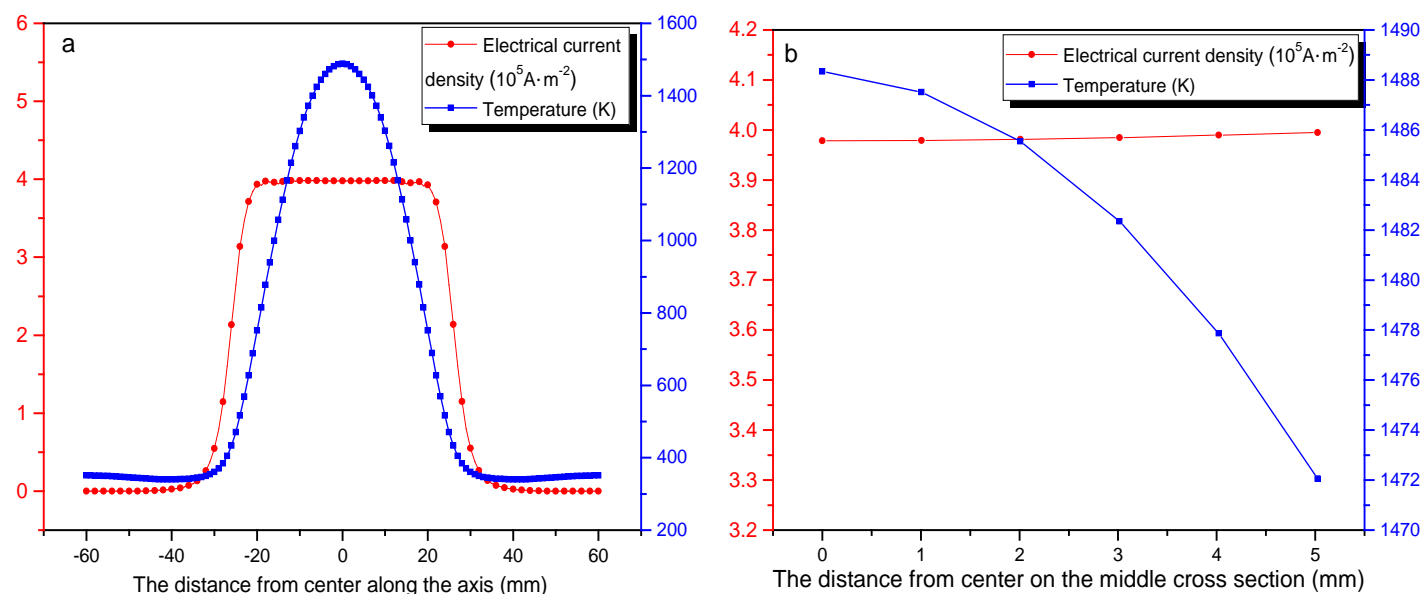

Fig. 4 The current densities and temperature distributions on the (a) axis and (b) middle cross section

As shown in the blue lines of Fig. 4, the temperature distribution in the axial direction is roughly normal distribution. The temperature on the $30 \mathrm{~mm}$ section of the specimen is stable at about $350 \mathrm{~K}$, but there is a very different on the middle section of $60 \mathrm{~mm}$. It can be know that the heat generated in the middle section is about equal 
from the current distributions. However, the two surfaces of the grips have continuous water cooling, so the heat inside always transmit from the center to the both grips, causing a big difference of the temperature in the axial. There are small differences in the temperature distribution on the cross section of the specimen too. The maximum difference is about $16 \mathrm{~K}$, when the test temperature is $1473 \mathrm{~K}$. The main reason for the temperature distribution on the cross section is the heat radiation of the specimen surfaces. During the heating process in actual test, because the temperature is always measured on the surface of specimen, which will make the internal temperature slightly higher than the ideal when the surface meets the test temperature.

\section{Effects of Temperature and Heat Preservation on Temperature Distributions}

As can be seen in Fig. 5. The results of simulation indicate that the trends of axial temperature distribution profiles are basically identical, but the profiles of the middle section in the specimen become steeper with the increase of the test temperature. The differences of sectional temperature distribution also become larger with increasing of test temperature. The maximum of differences is about $10 \mathrm{~K}$ when test temperature is $1273 \mathrm{~K}$, and about $23 \mathrm{~K}$ when $1673 \mathrm{~K}$. In general, the differences will increase of about $3 \mathrm{~K}$ with the test temperature rose of $100 \mathrm{~K}$.
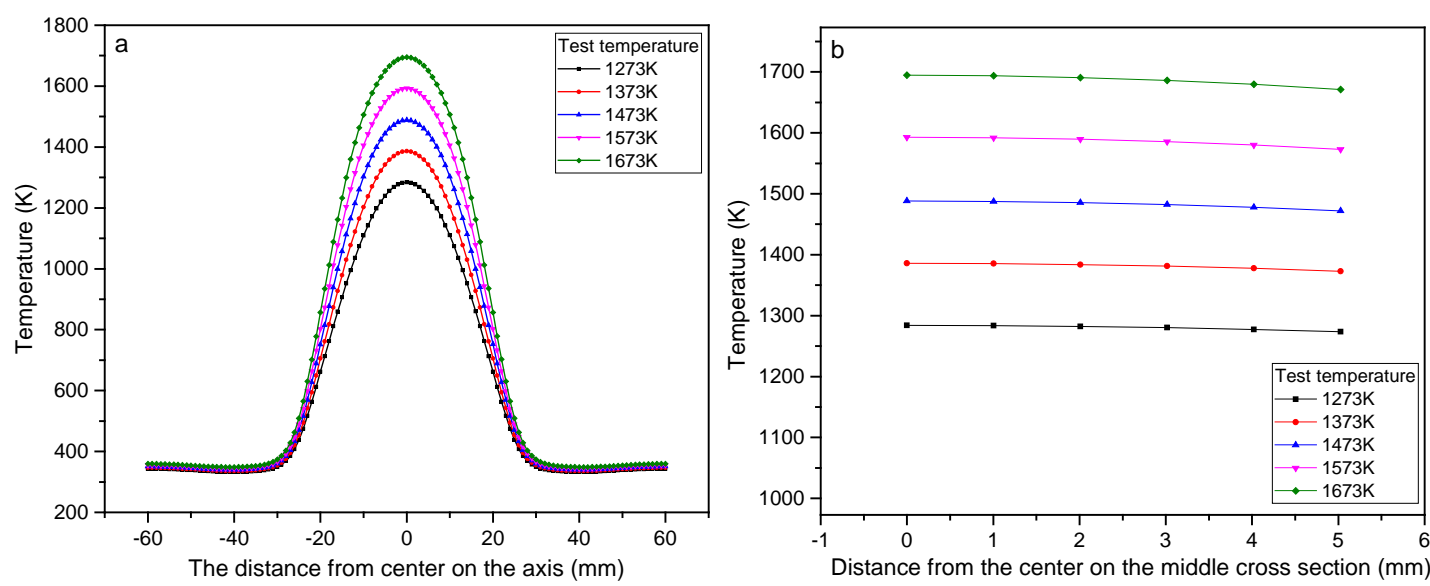

Fig. 5 The temperature distribution on the (a) axis and (b) middle cross section at different test temperatures

Because it is only a very small part of the center that can be exactly or approximately close to the desired test temperature. If the uniform temperature zone is allowable error $\pm 5 \mathrm{~K}$, then the lengths which meet the requirements are only about 4 $\mathrm{mm}$. However, the temperature outside uniform temperature zone is also relatively high, the deformation of the outside areas can not be ignored during tensile test. Otherwise, the strain obtained by the gauge length which set according to uniform temperature zone may be much larger than its true value in actual experiment.

Extending the time of heat preservation to $10 \mathrm{~min}$, there is almost no difference with the preserved of 2 min from the results in the test temperature of $1473 \mathrm{~K}$. Therefore, it was poor effectively attempting to improve the poor distributions of the internal temperature in the specimen by extending the time of heat preservation. 


\section{Conclusions}

1) After the specimens being heated to the test temperatures, the internal distributions are not uniform. The maximum temperature differences between the center and both ends are more than $1000 \mathrm{~K}$, and the maximum differences of the middle cross section maintained at about 10-20 K.

2) The uniform temperature zone is only about $4 \mathrm{~mm}$ within the temperature error of $\pm 5 \mathrm{~K}$. The difference on the section is not obvious with the increase of temperature, and the time of heat preservation is basically no effect on the temperature distributions.

3) In order to improve the test accuracy, it is possible to determine the gauge length by tensile simulation in stead of uniform temperature zone, or change the measurement of strain by measuring the lateral strain at a point on the surface of uniform temperature zone. In additional, it is a effective way by changing the shape of the specimen.

\section{Acknowledgments}

This work was financially supported by the National Natural Science Foundation of China (No. 51375041).

\section{References}

[1] Z.Deng, et al, Creep Properties of the Steel in the Rang of Temperature of Continuous Casting, Journal of University of Science \& Technology Beijing, 1991.

[2] X.H.Wang, G.Zhu, et al, High temperature properties of continuous casting high carbon steels, Journal of University of Science \& Technology Beijing. 27 (2005) 545-548.

[3] D.F.Chen, X.J.Gao, Q.M.Wang, et al. Research on high temperature mechanical property of Q235G slab, Chinese Journal of Process Engineering. 9 (2009) 210-213.

[4] D.J.Seol, Y.M.Won, et al, High Temperature Deformation Behavior of Carbon Steel in the Austenite and $\delta$-Ferrite Regions, ISIJ International. 39 (1999) 91-98.

[5] D.J.Seol, YM.Won, K.H.Oh, et al, Mechanical Behavior of Carbon Steels in the Temperature Range of Mushy Zone, ISIJ International. 40 (2000) 356-363.

[6] S.D.Norris, I.Wilson, Application of 3D numerical modeling for thermal profile optimization on the Gleeble thermomechanical simulator, Modeling \& Simulation in Materials Science \& Engineering. 7 (1999) 297-309.

[7] C.Zhang, M.Bellet, M.Bobadilla, et al, A Coupled Electrical-Thermal-Mechanical Modeling of Gleeble Tensile Tests for Ultra-High-Strength (UHS) Steel at a High Temperature, Metallurgical and Materials Transactions A. 9 (2010) 2304-2317. 